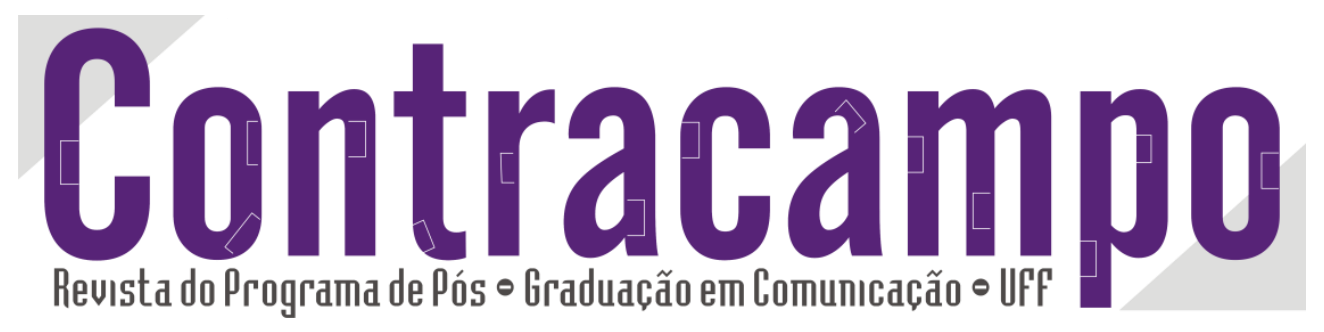

\title{
Negócios de audiovisual na internet: uma comparação entre Netflix, Hulu e iTunes-AppleTV, 2005-2010
}

Audiovisual business on the internet:

a comparison between Netflix, Hulu e iTunes-AppleTV, 2005-2010

João Martins Ladeira

joaomartinsladeira@gmail.com

Pesquisador associado aos grupos Unisinos Cepos e lesp Netsal

Ao citar este artigo, utilize a seguinte referência bibliográfica

LADEIRA, João Martins. Negócios de audiovisual na internet: uma comparação entre Netflix, Hulu e iTunesAppleTV, 2005-2010. In: Revista Contracampo, v. 26, n. 1, ed. abr, ano 2013. Niterói: Contracampo, 2013. Pags: 145-162

Enviado em: data de recebimento: 16 de ago. de 2012

Aceito em: data da aprovação: 08 de abr. de 2013

\section{PPGCOM UFF}

\section{Edição 26/2013}

Contracampo

Niterói (RJ), v. 26, n. 1, abr/2013.

www.uff.br/contracampo

A Revista Contracampo é uma revista eletrônica do Programa de PósGraduação em Comunicação da Universidade Federal Fluminense e tem como objetivo contribuir para a reflexão crítica em torno do campo midiático, atuando como espaço de circulação da pesquisa e do pensamento acadêmico. 


\section{Resumo}

O artigo analisa três novos negócios voltados para a convergência entre Internet e TV: Netflix, Hulu e Apple TViTunes. Partindo da economia política da comunicação, discute as disputas de poder num projeto que envolve intensa negociação entre grandes corporações de entretenimento e firmas menores. De um lado, antigos players da indústria cultural buscam intermediários capazes de garantir sua presença na web. De outro, empresas inovadoras tentam aumentar sua importância através de acesso a conteúdo com vasta aceitação. Demonstra-se que o streaming se configura não como uma experiência disruptiva, mas conectada a um antigo mecanismo institucional da indústria cultural: a administração sobre os direitos de para distribuição de conteúdo.

Palavras-chave: Convergência Digital, Economia Política da Comunicação, inovação, Empreendedorismo.

\section{Abstract}

This article analysis three new business oriented towards the convergence between internet and television: Netflix, Hulu and Apple TV-iTunes. Adopting as a reference the political economy of communication, it discusses the power struggles in a project characterized by the intense negotiation between huge entertainment corporations and smaller firms. On one hand, old players seek intermediaries capable to warrant their presence on the web. On the other, innovative enterprises try to enhance their importance towards the access to content with wide acceptation. The article demonstrates that the streaming configures itself not as a disruptive experience, but as a consequence of an old institutional mechanism from the cultural industry: the administration of content distribution rights.

Keywords: Digital Convergence, Political Economy of Communication, Innovation, Entrepreneurship. 
$\mathrm{E}$ ste artigo analisa uma fração específica do processo de convergência entre TV e internet, com foco no nascente segmento de distribuição, através de firmas pontocom laterais ao mercado audiovisual, de conteúdo criado não por usuários amadores, mas pelos estúdios e redes de televisão. Discutem-se três empreendimentos, Netflix, Hulu e iTunes-AppleTV, abordando o período entre 2005 e 2010. Embora possibilidades para difundir conteúdo na rede se tornem visíveis após o aprimoramento do YouTube, tal produto se volta, desde sua concepção, para usuários amadores, sem atenção a formas economicamente viáveis para veicular conteúdo premium. Na busca por alternativas para comercializar tanto os imensos acervos quanto as recentes criações de grandes produtores, Netflix, Hulu e iTunes-AppleTV delimitam projetos de negócios capazes de reunir grupos tradicionais de comunicação e firmas de internet, num caso de alianças estratégicas e de cooperação entre grandes e pequenas firmas.

A economia política da comunicação, orientação focada em compreender a organização institucional da mídia, especialmente a lógica de propriedade dos meios e as consequências de cada ordenamento de posse (Golding, Murdock, 1996; Bustamante, 2003), dedicou especial atenção à reorganização das indústrias culturais contemporâneas, no jornalismo (Albornoz, 2007), na indústria da música (Herschmann, 2007) ou no rádio (Kischinhevsky, 2007). No setor audiovisual (Bolaño, Brittos, 2007), debateram-se as tendências para produção de conteúdo, concentração de propriedade e desregulamentação de mercados. Compreender a apropriação de novas oportunidades significa entender a lógica da reorganização do sistema capitalista. Trata-se de um estudo sobre o funcionamento de mercados, analisando sua operação como consequência não apenas de fatores econômicos, mas também de negociações de poder entre personagens com diferentes capacidades para controlar recursos, numa luta cujo 
resultado é a definição de normas e modelos de operação neste espaço de relações sociais.

Os casos trabalhados buscam formas comercialmente viáveis de negociar, na rede, filmes e séries. Nenhum modelo decisivo ou solução paradigmática parece ter sido encontrado, indicando um processo em aberto. $\mathrm{Na}$ verdade, este texto descreve uma série contraditória de ações: o cuidado das grandes corporações em defender seu conteúdo e, ao mesmo tempo, a tentativa de novos negócios se afirmarem recorrendo a acordos que permitam a utilização de material já conhecido pelo público. Este novo mercado envolve o relacionamento entre velhos personagens, grupos tradicionais de comunicação em busca da manutenção de seu poder, e empresas voltadas à internet construindo inovações. A aliança entre ambos indica uma relação de mútua dependência, envolvendo uma duplicidade: sem conteúdo, os novos empreendimentos podem se tornar inviáveis; sem a presença na web, as antigas corporações deixam escapar a chance de participar de um novo mercado. Depara-se, assim, com um momento no qual ambos se veem obrigados a negociar.

A tensão resultante decorre de esforço de definir qual lugar o streaming pode ocupar frente ao restante da indústria. Neste artigo, abandona-se a expectativa sobre a transformação radical, a partir da convergência entre televisão e internet, da organização já estabelecida para o mercado. Ao invés de esperar uma renovação decisiva no modelo de negócio próprio ao setor, defende-se a delimitação da trajetória a ser adotada como um processo negociado. Entender a diferença de poder em tais acertos e a forma pela qual alguns tentam suplantá-la e outros, mantê-la, torna-se o tema do artigo.

Aqui, interpreta-se a necessidade de explorar a reutilização de produtos em distintos canais como uma das principais estratégias do setor audiovisual (Turow, 2003). Em geral, e também para os negócios de streaming em particular, películas e conteúdos de mesmo tipo possuem uma hierarquia estabelecida através da rapidez com a qual se permite seu acesso ao público. O tempo de espera artificialmente criado permite atribuir valores diferenciados, como se fossem produzidas versões de uma mesma mercadoria: o lançamento no cinema; a distribuição em DVDs; o licenciamento para televisão a cabo ou satélite; a distribuição para TVs abertas; tais eram as etapas existentes. Para o streaming, a questão se torna delimitar um lugar neste antigo circuito. 
Para as empresas que oferecem o serviço, valorizá-lo significa disponibilizar material inédito imediatamente acessível ao público. Para os produtores de conteúdo, continua a ser importante proteger as vendas de DVDs e os investimentos em TV por assinatura, sem deixar de afirmar sua presença na internet. Assim, retardar a velocidade com que o streaming utiliza conteúdo possui importância não desprezível. Entendem-se tais associações entre empreendimentos visando certo projeto de negócios como empresas em rede (Castells, 2001). Tal questão adiciona o desafio de discutir o tipo de associação necessária entre negócios variados a fim de levar a cabo um empreendimento específico.

Este texto se divide da seguinte forma: a primeira parte apresenta o debate sobre concentração industrial e as sinergias possíveis de obter. Frisa-se como a intensa diferenciação dos meios de comunicação permite a novas firmas participarem em brechas de mercado ainda inexploradas. A descrição das estratégias adotadas ocorre na seção seguinte. As negociações com grandes corporações de conteúdo são o tema da última parte. A conclusão problematiza a sobrevivência de tais firmas, especialmente sua capacidade de resistir à internalização logo que uma trajetória consistente for definida.

\section{Concentração, sinergia e convergência: os meios de comunicação contemporâneos}

A concentração dos meios após os anos 80 e a oportunidade oferecida para grandes grupos racionalizarem de modo intenso a exploração do mercado se constitui em tema já tratado pela economia política da comunicação. Indicou-se de que modo redefinir a dispersão entre as muitas atividades (produção de notícias, entretenimento, fonogramas, audiovisual, conteúdo radiofônico) a partir de uma integração bastante forte apontou para a exploração das assim chamadas sinergias, oportunidade de reutilizar o mesmo conteúdo, comercializando-o em formatos diferentes (Mosco, 2009).

Tal noção de sinergia consiste em explorar todas as possibilidades de um mesmo bem, reaproveitando-o em mídias distintas. Assim, problemas intrínsecos à negociação de direitos de propriedade ou à organização de estratégias de divulgação, entre outros, podem ser mais racionalmente administrados. Torna-se possível planejar formas de licenciar o mesmo produto com as transformações necessárias para adaptá-lo 
a novos meios, como no exemplo da transposição de livros para o cinema. Do mesmo modo, acordos de licenciamento de conteúdo assumem relevância específica, por permitirem novos ganhos sem transformações na mercadoria original: a venda de filmes para televisão empreendida por estúdios e produtoras exemplificam este movimento (Murray, 2005; Wasko, 2006).

Todavia, tal processo de concentração foi não linear. Grandes corporações passaram não apenas a reunir negócios distintos, mas, também, a criar subdivisões administrativas dentro delas mesmas, permitindo lidar com as especificidades dos negócios internalizados. Pode-se, assim, obter competição entre diversos segmentos. Permite-se a canais exclusivos de all-news ou de esportes disputar público através de estratégias pontuais, embora permaneçam associados a grandes corporações (Doyle, 2010). Tal organização da indústria encontra na digitalização uma oportunidade técnica relevante. Afinal, como amplamente destacado, tal inovação permitiu transportar com facilidade material entre meios, oferecendo velocidade à reapropriação.

Porém, tal diferenciação possui duplo efeito. Por um lado, proporciona à grande corporação flexibilidade para explorar múltiplos segmentos. Por outro, permite novos personagens surgirem nas brechas deixadas por nichos ainda não explorados. Usualmente, este caso envolve a entrada de novas firmas em atividade. Devido à estrutura de poder do setor, poucos empreendimentos possuem a capacidade de atuar de modo autônomo, dispensando associações com outras empresas para se manter. Entende-se a interdependência entre diversas corporações visando determinados projetos de negócios como característica social contemporânea decorrente da ampliação da complexidade, identificada por Castells (2001) pelo rótulo de empresa em rede.

Solução atual para a organização econômica através da associação de firmas atuando numa economia de dimensão global, a empresa em rede descreve um cenário no qual, ao invés de internalizar as competências necessárias para a execução de determinadas tarefas, dispersam-se tais atividades em subcontratos, buscando flexibilidade e velocidade capaz de criar produtos diversificados. Grandes e pequenas firmas têm de se adequar ao formato, transformando a organização em rede num poderoso instrumento, sem implicar na redução de importância das corporações de maior dimensão. 
As redes formadas por grandes corporações de conteúdo e firmas pontocom buscam o licenciamento de material capaz de permitir às empresas citadas operarem como intermediários (Marchi, Albornoz, Herschmann, 2011), distribuindo bens de variados produtores e transformando tais projetos em atividades possíveis de envolver múltiplos agentes. O tema da sinergia, que surge acompanhada de um tipo de concentração capaz de comportar a fragmentação e diferenciação dos meios de comunicação, passa, a seguir, a ser observado nos três empreendimentos de interesse.

\section{Novos negócios: Netflix, Hulu, Apple TV-iTunes}

As firmas aqui tratadas representam uma experiência de empreendedorismo, introduzindo novos negócios num mercado. Sua contribuição reside em desenvolver estratégias que permitam agir num ambiente no qual antigas corporações possuem extrema relevância e intenso poder sobre a mercadoria indispensável à sobrevivência das novas empresas devido ao controle exercido através de normas de propriedade intelectual. A velocidade com que estes empreendimentos estabeleceram uma atividade com significativo interesse do público - a capacidade de receber audiovisual através da web - associada à resistência dos proprietários de conteúdo em desenvolver eles mesmos uma alternativa viável, envolve ambos num mesmo projeto.

Como distribuidores de material licenciado, cada um tem soluções particulares de intermediação. Netflix oferece um serviço de assinaturas; Hulu começa como atividade gratuita financiada pela publicidade, aderindo posteriormente ao modelo de seu concorrente; Apple TV-iTunes se centra na rede de bens controlada pela própria Apple. Seus históricos frisam as distinções afirmadas por suas escolhas. O interesse em descrevê-los reside em indicar como cada um institui poder e capacidade de negociação na rede em que opera.

\section{Netflix}

Em operação desde 04/1998, o Netflix experimentou diversos modelos de atuação até adotar o streaming. Inicialmente, organizou-se como serviço para compra e aluguel de DVDs em atividade apenas nos EUA. Estabelecido como negócio online, realiza-se os pedidos pela internet e o envio/retorno da encomenda, pelo correio. Misto 
de loja e locadora, encerra a venda de discos em 03/1999 e inaugura em 09/1999 o serviço de assinaturas. Este segundo modelo permite ao consumidor uma quantidade fixa de remessas mensais e, à firma, um fluxo constante de recursos, ao invés da venda unitária de produtos. Assim, posiciona-se frente aos criadores de conteúdo como personagem habilitado na venda de bens com posição clara na hierarquia do setor: os DVDs. A vantagem do Netflix frente a locadoras convencionais está no tamanho do catálogo e na extensão geográfica alcançada através da remessa pelos correios. Em 2002, o número de títulos era de aproximadamente 14.500 itens, enviados através dos 18 centros de distribuição nos EUA, permitindo atingir com velocidade diversos pontos do país. Com tal modelo, a empresa abre capital em 04/2002 (SEC, 2002).

A transformação posterior vem em 2007, ao inaugurar o serviço de streaming (SEC, 2007a). A partir de 11/2010, o Netflix estreia um plano específico para acesso a audiovisual na rede, dispensando a remessa de DVDs (Kopytoff, 2010a) e indicando maior atenção ao mercado online. Todavia, no terceiro trimestre de 2010, um total de 58 pontos de distribuição possuíam contratos com Netflix (Kopytoff, 2010b), acordos que se encerrariam apenas em 2016 (SEC, 2010), tornando difícil crer na possibilidade da empresa abandonar o modelo de remessa de discos de modo tão rápido.

A despeito da atuação mais intensa na internet, seus negócios ainda se restringem aos EUA. Somente em 09/2010 expande sua atuação para o Canadá, distribuindo audiovisual naquele país através apenas da web (Kopytoff, 2010b). Curiosa contradição: negócio online com potencial ilimitado de difusão, a atividade se restringe a fronteiras nacionais, devido à complexidade de negociar direitos de propriedade em escala mundial.

\section{Hulu}

Diferente do Netflix, o Hulu surge como negócio online. Instituído em 10/2007 como um experimento atendendo a um número limitado de usuários, estreia em 03/2008, embora restrito aos EUA (Hulu.com Opens to Public , 2008). Empreendimento conjunto entre NBC e News Corp, conta com financiamento de US\$ 100 milhões do fundo de investimento especializado em meios de comunicação Providence Equity Partners, que adquire 10\% da firma em 08/2007 (Stone, 2007a). Em 05/2009, a ABC 
compra $28 \%$ do Hulu, oferecendo crédito publicitário de US\$ 25 milhões na rede de televisão (Stelter, Stone, 2009).

Desde o princípio, o Hulu surge como uma aliança estratégica entre corporações tradicionais de audiovisual. Sua criação faz parte de um projeto a partir do qual vários personagens se ligam com o objetivo de permitir a distribuição de algum conteúdo através da rede, sem, todavia, nenhum tomar a frente do projeto. Na verdade, o Hulu opera com uma administração autônoma, na qual todos os fracassos podem ser atribuídos à firma, enquanto os sucessos funcionam como aprendizado sobre os rumos a tomar.

Inicialmente, o serviço depende da receita de anunciantes. Os fornecedores de conteúdo recebiam entre $50 \%$ e $70 \%$ da publicidade gerada pelo serviço (Stelter, Stone, 2010). A negociação sobre valores foi sempre tema complexo. A Viacom, por exemplo, chega a retirar material em 03/2010 devido a dificuldades de acordo (Stelter, 2010a). O fato do Hulu ser propriedade de redes de televisão torna outras negociações igualmente difíceis. Durante bom tempo, o serviço oferece acesso apenas através de computadores, na tentativa de evitar a competição com a televisão paga. Num outro exemplo, em 02/2009 a firma corta, a pedido dos fornecedores de conteúdo, a distribuição para o Boxee, software que permite assistir a conteúdo em televisores (Stone, 2009). O limite à exibição através de computadores se encerra com o lançamento em 06/2010 de um serviço premium, o Hulu Plus, garantindo um segundo tipo de rendimento.

\section{iTunes-AppleTV}

A estratégia de Apple a difere de seus concorrentes. As características da corporação estão ai presentes. Firma independente, e não uma aliança criada para tal projeto, distingue-se por entrar no negócio de difusão de conteúdo devido ao amadurecimento do mercado de desktops, tendência percebida desde o investimento em seu equipamento próprio para reprodução de áudio, o iPod. Logo, a atenção à necessidade de reunir a comercialização do equipamento eletrônico à distribuição de conteúdo surge como problema essencial.

Traços utilizados durante a experiência com venda de música foram reapropriados neste segundo momento. A importância à rede de produtos administrada pela Apple surge como ponto essencial. Em 03/2007, a corporação disponibiliza um 
serviço capaz de permitir a recepção de conteúdo em aparelhos televisores, o Apple TV (SEC, 2007b). Embora oferecesse acesso a material distribuído por Netflix, YouTube, Flickr e MobileMe, sua diferença reside na associação com serviços proprietários administrados pela própria Apple. Na verdade, o set-top box se conectava diretamente à loja iTunesStore, primeira opção para compra de material, oferecendo outra dinâmica ao serviço.

Este modelo começou a ser delimitado em 2001 com a comercialização dos iPods. Inicialmente um hardware com conteúdo executado como num walkman digital, transforma-se posteriormente num instrumento capaz de permitir a venda de música através da loja online, o iTunesStore, em operação desde 04/2003 (SEC, 2009). Completando o conjunto de bens complementares que envolve equipamento e conteúdo, este último serviço se associa ao software para execução de arquivos de áudio iTunes.

Explica-se tal elo pela tentativa de travar o consumidor tanto ao conteúdo quanto ao equipamento da Apple, preocupação ausente em outros serviços. Embora também dependa da associação com produtores tradicionais de entretenimento, sua opção foi internalizar o nó relativo ao design e à comercialização do equipamento, afirmando-o como um complemento capaz de expandir o consumo de conteúdo. A próxima seção indica como os novos negócios negociam a fim de reorganizar as janelas para lançamento de material, na tentativa de afirmar sua importância frente ao modelo de licenciamento em prática.

\section{Corporações de audiovisual e proteção de conteúdo}

Frente ao cenário de concentração e sinergia, a questão relevante se refere ao intervalo para licenciar um produto em diferentes formatos. Corporações verticalmente integradas definiriam tal intervalo unilateralmente: possivelmente, grandes grupos restringiriam o streaming à distribuição de acervos, sem interferir no conteúdo inédito. Como empreendimentos autônomos, porém, duas destas firmas não se encontram integradas às corporações tradicionais de entretenimento e uma delas foi criada buscando exatamente a autonomia para tais acertos. Logo, tal relação demanda negociações nas quais as diferenças de poder e a apropriação de oportunidades 
produzem um equilíbrio tenso de variados acordos. A tabela abaixo indica o primeiro momento em que conteúdo é licenciado para cada um delas.

Tabela 1: Acordos iniciais de licenciamento entre firmas de streaming e estúdios/redes

\begin{tabular}{|c|c|c|c|}
\hline $\begin{array}{lr} & \text { Corpor } \\
\text { ações } & \text { de } \\
\text { conteúdo }\end{array}$ & etflix & ulu & Tunes \\
\hline $\mathrm{ABC}$ & ez/10 & $\mathrm{ul} / 09$ & ut/05 \\
\hline CBS & d. & d. & ar/06 \\
\hline Fox & $\mathrm{br} / 10$ & ut/07 & ai/06 \\
\hline MGM & d. & ut/07 & d. \\
\hline $\mathrm{NBC}$ & ov/07 & ut/07 & $\mathrm{ez} / 05$ \\
\hline Sony & d. & ut/07 & d. \\
\hline Warner & an/10 & ar/08 & .d. \\
\hline
\end{tabular}

Fonte: elaboração própria

Os acordos envolvem filmes e séries, deixando de lado produtos como, por exemplo, o jornalismo. Desta forma, restringe-se o tipo de regulamentação a definir. Um negócio baseado na difusão de notícias possuiria uma tolerância diferente em relação a material antigo. No caso da ficção, os intervalos podem ser mais extensos, sem deixar de conter interesse. A principal restrição estava na imposição de Netflix, Hulu e Apple respeitarem o intervalo para lançamento de DVDs, primeiro e mais lucrativo espaço. A partir dai, o tema seria definir o lugar ocupado em relação a serviços de payper-view e televisão a cabo e satélite.

A Apple é a primeira a negociar acordos para difusão de audiovisual. Os contratos com ABC, NBC, Viacom e Fox, em 2005 e 2006, voltados inicialmente para 
distribuição de séries, envolvem 51 títulos (Apple Announces iTunes..., 2005; NBC Universal \& Apple..., 2005; MTV Networks' Hit ..., 2006; Hit Programming from Fox , 2006). Este seria o foco até a associação com Lionsgate em 02/2007 para a distribuição de filmes. Em 03/2007, vem o lançamento de seu set-top box. Em 01/2008, a Apple disponibiliza um serviço conhecido como iTunes Movie Rentals, oferecendo uma espécie de pay-per-view, com taxas cobradas por filme locado, envolvendo um intervalo máximo para assistir o material (SEC, 2009). O iTunes Movie Rentals distribuía conteúdo com uma janela de 30 dias após o lançamento do DVD. No lançamento, o acervo do serviço era pequeno, com aproximadamente 1.000 títulos (Apple Premieres iTunes..., 2008).

O Hulu, associação entre duas corporações de conteúdo, teria garantias de acesso a catálogos de NBC e News, com os programas da ABC distribuídos meses após o acordo entre ambas (Hulu Starts Showing , 2009). Ao mesmo tempo, garantem-se acordos com MGM e Sony desde o início (Hulu Pulls Back..., 2007). Por outro lado, a resposta da NBC em relação a seu relacionamento com YouTube e Apple indicam a tensão criada ao defender o serviço. Em 10/2007, a NBC remove conteúdo e fecha um canal promocional do YouTube (Stone, 2007b). Em 09/2007, impõe novas exigências de preço à Apple, não aceitas (NBCU Declines to Renew..., 2007). O conteúdo retornaria ao iTunes apenas em 09/2008 (NBC Universal Returns , 2008).

Ao entrar no negócio de streaming, o Netflix busca, a despeito dos acordos com redes e estúdios, criar relações também com distribuidoras e canais de televisão por assinatura. Afinal, o acervo inicialmente licenciado para streaming foi de material bastante antigo. Em 10/2008, os contratos de distribuição ganham fôlego através do acordo com Starz, serviço de TV paga de propriedade da Liberty Media. O acerto prevê acesso a 2.500 títulos de propriedade da Disney e Sony (Netflix and Starz Entertainment..., 2008). A Starz já buscara, no passado, modelos de distribuição via web semelhantes aquele estabelecido com Netflix, mantendo-se mais ativo que outros concorrentes neste quesito (Harmon, 2002; Gnatek, 2004; Hansell, 2006).

Em 08/2010, Netflix fecha acordo com Epix, canal de TV por assinatura de propriedade de Paramount, MGM e Lionsgate, mas sem distribuição por serviços de transmissão via satélite de importância. Não se divulga o valor do acordo, mas estima-se o pagamento de US\$ 900 milhões por cinco anos (Stelter, 2010b; Arango, Stelter, 
2009). Do mesmo modo, efetiva dois acordos com estúdios: Relativity Media, em 07/2010, e Millennium Films, em 09/2010 (Netflix and Relativity Media..., 2010; Netflix and Nu Image/Millennium Films..., 2010), prevendo distribuição de material 90 dias após a estréia em TVs por assinatura, preservando uma janela, mas abrindo novas possibilidades para a firma encurtar intervalos. Logo, enquanto a Apple dá início ao processo de licenciamento com séries, Hulu e Netflix desenvolvem modelos de assinaturas para filmes e, progressivamente, todos os três empreendimentos irão oferecer atenção a ambos os produtos.

\section{Conclusão}

Os serviços de streaming pela internet bem poderiam ser compreendidos como uma alternativa em choque com negócios já estabelecidos de audiovisual, especialmente os empreendimentos de TV por assinatura. Porém, a sequência de acordos entre redes, produtoras e distribuidoras de filmes e séries torna difícil aceitar esta interpretação. Outra leitura apresenta o streaming como uma forma de criar novos elementos na cadeia que compõe o ciclo de produtos do setor audiovisual.

Deste modo, compreendem-se tais serviços como formas de explorar capacidades detectadas por determinados empreendedores. Para eles, a principal questão está em reutilizar um velho modelo organizacional, baseado na criação de versões que permitam a diferenciação de produtos de comunicação. No caso do audiovisual, as etapas de distribuição envolvem a exibição em salas de cinema, o licenciamento para o mercado de DVDs, a autorização para difusão em serviços de televisão por assinatura, a liberação para TVs abertas. Os serviços de streaming, ao invés de romper esta cadeia, constituem-se como outra possibilidade para licenciamento e difusão.

Todavia, considerando a extensa consolidação das últimas décadas, serão os negócios de streaming um experimento a ser internalizado por grandes corporações após se identificar modelos viáveis de negócios? Sua dependência em relação aos produtores de conteúdo os coloca numa situação delicada. O cenário espelha um traço típico à organização da indústria de comunicação, discutido há décadas pela economia política: o intenso poder proporcionado a tais corporações através do controle de conteúdo. De qualquer modo, a importância de títulos conhecidos contém mais relevância que 
renovações tecnológicas por si mesmas. Tal indica a possibilidade da inovação técnica ter, neste setor, importância muito menor que discussões sobre "novas tecnologias" atribuíram.

\section{Referências bibliográficas}

ALBORNOZ, L. Periodismo Digital: Los Grandes Diarios en la Red. Buenos Aires: La Cruja, 2007

Apple Announces iTunes 6 With 2,000 Music Videos, Pixar Short Films \& Hit TV Shows. Apple, 12 out. 2005. Disponível em: http://www.apple.com/pr/library/2005/oct/12itunes.html. Acesso em: 09 fev. 2011

Apple Premieres iTunes Movie Rentals With All Major Film Studios. PR Newswire, 15 jan, 2008.

Disponível

em:

http://go.galegroup.com/ps/i.do?id=GALE\%7CA173435969\&v=2.1\&u=capes2\&it=r\&p=AON $\underline{E \& s w=w}$. Acesso em 18 jan. 2011

ARANGO, T.; STELTER, B. Film Channel Has Name, But No Network Carrier. The New York Times, p. B2(L), 28 jan. 2009. Disponível em: http://go.galegroup.com/ps/i.do?id=GALE\%7CA192662148\&v=2.1\&u=capes2\&it=r\&p=AON E\&sw=w. Acesso em 1 fev. 2011

BOLAÑO, C; BRITTOS, V. A Televisão Brasileira na Era Digital. São Paulo: Paulus, 2007

BUSTAMANTE, E. (org). Hacia un Nuevo Sistema Mundial de Comunicación. Barcelona: Gedisa, 2003

CASTELLS, M. Rise of the network society. Oxford: Blackwell, 2001.

DOYLE, G. From Television to Multi-Platform: Less from More or More for Less. Convergence v. 16, n. 4, p. 431-49, 2010

EPIX and Netflix Announce Exclusive Internet-Only Deal to Instantly Stream EPIX Movies to Netflix Members. ENP Newswire, 11 ago. 2010. Disponível em: http://go.galegroup.com/ps/i.do?id=GALE\%7CA234163348\&v=2.1\&u=capes2\&it=r\&p=AON E\&sw=w. Acesso em: 10 jan. 2011

GNATEK, T. An Online Supplier for Your Desktop Cineplex. The New York Times, p. G8, 12 ago. 2004.

Disponível em: http://go.galegroup.com/ps/i.do?id=GALE\%7CA120506450\&v=2.1\&u=capes2\&it=r\&p=AON E\&sw=w. Acesso em: 1 fev. 2011

GOLDING, P.; MURDOCK, G. Culture, Communication and Political Economy. CURRAN, J.; GUREVITCH, M. (org.). Mass Media and Society. London: Arnold, 1996.

HANSELL, S. Provider of Movies on Cable Looks To Expand to PC's and Video Players. The New York Times, p. C1(L), 3 jan. 2006. Disponível em: http://go.galegroup.com/ps/i.do?id=GALE\%7CA140430985\&v=2.1\&u=capes2\&it=r\&p=AON E\&sw=w. Acesso em: 1 fev. 2011 
HARMON, A. Technology Briefing Internet: Movie Subscription Service Is Set To Begin. The New York Times, p. C11, 4 dez. 2002. Disponível em: http://go.galegroup.com/ps/i.do?id=GALE\%7CA94851313\&v=2.1\&u=capes2\&it=r\&p=AONE $\underline{\& s w=w}$. Acesso em: 1 fev. 2011.

HERSCHMANN, M. Lapa: Cidade da Música. Rio de Janeiro: Mauad, 2007

Hit Programming from Fox Entertainment Group's Fox, FX, Speed, Fuel \& 20th Century Fox Television Library Now Available on the iTunes Music Store. Apple, 9 mai. 2006. Disponível em: http://www.apple.com/pr/library/2006/may/09fox.html. Acesso em: 09 fev. 2011

Hulu Pulls Back Curtain, Privately. Multichannel News, v. 28, n. 44, p. 28, 5 nov. 2007. Disponível em: http://go.galegroup.com/ps/i.do?id=GALE\%7CA170726328\&v=2.1\&u=capes2\&it=r\&p=AON $\underline{E \& s w}=\mathrm{w}$. . Acesso em: 14 jan. 2011

Hulu Starts Showing ABC Content. PC Magazine Online, 6 jul. 2009. Disponível em: http://go.galegroup.com/ps/i.do?id=GALE\%7CA203178688\&v=2.1\&u=capes2\&it=r\&p=AON $\underline{E \& s w=w}$. Acesso em: 14 jan. 2011

Hulu.com Opens to Public, Offers Free Streams of Hit TV Shows, Movies and Clips from More Than 50 Providers Including FOX, NBC Universal, Metro-Goldwyn-Mayer Studios Inc. and Sony Pictures Television, Hulu, 12 mar. 2008. Disponível em: http://www.hulu.com/press/launch_press_release.html. Acesso em: 31 jan. 2011.

KISCHINHEVSKY, M. O rádio sem onda: convergência digital e novos desafios da radiodifusão. Rio de Janeiro: E-Papers, 2007

KOPYTOFF, V. G. A Cheaper Plan at Netflix Offers Films for Online Only. The New York Times, p. B2(L), 23 nov. 2010a. Disponível em: http://go.galegroup.com/ps/i.do?id=GALE\%7CA242640845\&v=2.1\&u=capes2\&it=r\&p=AON E\&sw=w. Acesso em: 20 jan. 2010

KOPYTOFF, V. G. Shifting online, Netflix faces new competition. The New York Times, p. B1(L), 27 set. 2010b. Disponível em: http://go.galegroup.com/ps/i.do?id=GALE\%7CA238066722\&v=2.1\&u=capes2\&it=r\&p=AON E\&sw=w. Acesso em: 20 jan. 2010

MARCHI, L, ALBORNOZ, L, HERSCHMANN, M. Novos negócios fonográficos no Brasil e a intermediação do mercado digital de música. Famecos, v. 18, p. 279-291, 2011.

MOSCO, V. The Political Economy of Communication. London: SAGE Publications, 2009.

MTV Networks' Hit Programming from MTV, COMEDY CENTRAL \& Nickelodeon Now Available on the iTunes Music Store. Apple, 26 jan. 2006. Disponível em: http://www.apple.com/pr/library/2006/jan/26itms.html. Acesso em: 09 fev. 2011

MURRAY, S. Brand loyalties: rethinking content within global corporate media. Media, Culture \& Society, v. 27, n. 3, p. 415-435, 2005 
NBC Universal \& Apple Offer New Primetime, Cable, Late-Night \& Classic TV Shows on the iTunes Music Store. Apple, 6 dez. 2005. Disponível em: http://www.apple.com/pr/library/2005/dec/06nbc.html. Acesso em: 09 fev. 2011

NBC Universal Returns to the iTunes Store. PR Newswire., 9 set. 2008. Disponível em: http://go.galegroup.com/ps/i.do?id=GALE\%7CA184653413\&v=2.1\&u=capes $2 \& i t=r \& p=A O N$ E\&sw=w. Acesso em: 19 jan. 2011

NBCU Declines to Renew iTunes Contract. Broadcasting \& Cable, v. 137, n. 35, p. 22, 3 set. 2007.

http://go.galegroup.com/ps/i.do?id=GALE\%7CA168316881\&v=2.1\&u=capes2\&it=r\&p=AON $\underline{E \& s w=w}$. Acesso em: 14 jan. 2011

Netflix and $\mathrm{Nu}$ Image/Millennium Films Announce Long-term Agreement to Stream First-Run Theatrical Films to Netflix Members. PR Newswire, 8 set. 2010. Disponível em: http://go.galegroup.com/ps/i.do?id=GALE\%7CA236635838\&v=2.1\&u=capes2\&it=r\&p=AON E\&sw=w. Acesso em: 10 jan. 2011

Netflix and Relativity Media Announce Groundbreaking Deal to Stream First Run, Studio Quality Theatrical Movies to Netflix Subscribers. PR Newswire, 6 jul. 2010. Disponível em: http://go.galegroup.com/ps/i.do?id=GALE\%7CA230741603\&v=2.1\&u=capes2\&it=r\&p=AON E\&sw=w. Acesso em: 10 jan. 2011

Netflix and Starz Entertainment Announce Agreement to Make Movies From Starz Play Available for Instant Streaming at Netflix. PR Newswire, 1 out. 2008. Disponível em: http://go.galegroup.com/ps/i.do?id=GALE\%7CA186114316\&v=2.1\&u=capes2\&it=r\&p=AON E\&sw=w. Acesso em: 10 jan. 2011

SEC. Apple, Inc: Annual Report. Washington, D.C: US SEC, 2007b

SEC. Apple, Inc: Annual Report. Washington, D.C: US SEC, 2009

SEC. Netflix, Inc: Annual Report. Washington, D.C: US SEC, 2002

SEC. Netflix, Inc: Annual Report. Washington, D.C: US SEC, 2007a

SEC. Netflix, Inc: Annual Report. Washington, D.C: US SEC, 2010

STELTER, B. Viacom And Hulu Part Ways. The New York Times, p. B1(L), 3 mar. 2010a. Disponível em: http://go.galegroup.com/ps/i.do?id=GALE\%7CA220114940\&v=2.1\&u=capes2\&it=r\&p=AON E\&sw=w. Acesso em: 19 jan. 2011

STELTER, B. Netflix to Pay Nearly \$1 Billion to Add Films to On-Demand Service. The New York Times, p. B3(L), 11 ago. 2010b. Disponível em: http://go.galegroup.com/ps/i.do?id=GALE\%7CA234116933\&v=2.1\&u=capes $2 \& i t=r \& p=A O N$ E\&sw=w. Acesso em: 20 jan. 2010

STELTER, B.; STONE, B. ABC to Add Its Shows To Videos On Hulu. The New York Times, p. B7(L), 5 jan. $2009 . \quad$ Disponível em: http://go.galegroup.com/ps/i.do?id=GALE\%7CA198830136\&v=2.1\&u=capes2\&it=r\&p=AON $\underline{E \& s w=w}$. Acesso em: 19 jan. 2011 
STElTER, B.; STONE, B. Successes (and Some Growing Pains) at Hulu. The New York Times, p. $\quad$ B1(L), 1 abr. 2010. Disponível em: http://go.galegroup.com/ps/i.do?id=GALE\%7CA222648961\&v=2.1\&u=capes2\&it=r\&p=AON E\&sw=w. Acesso em: 19 jan. 2011

STONE, B. Equity firm invests in NBC Universal-News Corp. Online Venture. The New York Times, p. C3(L), 9 ago. 2007a. Disponível em: http://go.galegroup.com/ps/i.do?id=GALE\%7CA167372474\&v=2.1\&u=capes2\&it=r\&p=AON E\&sw=w. Acesso em: 19 jan. 2011

STONE, B. Hulu readies its online TV, dodging the insults. The New York Times, p. C1(L), 29 out. 2007b. Disponível em: http://go.galegroup.com/ps/i.do?id=GALE\%7CA170361959\&v=2.1\&u=capes2\&it=r\&p=AON

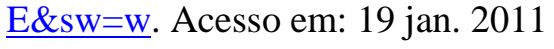

STONE, B. Hulu Will Cut Off Users of Boxee. The New York Times, p. B4(L), 23 fev. 2009. Disponível http://go.galegroup.com/ps/i.do?id=GALE\%7CA194258772\&v=2.1\&u=capes2\&it=r\&p=AON E\&sw=w. Acesso em: 19 jan. 2011

TUROW, J. Media Today: An Introduction to Mass Communication. London. Routledge, 2003.

WASKO, J. Understanding the Disney Universe . CURRAN, J.; GUREVITCH, M. (org.). Mass Media and Society. London: Arnold, 1996. 